\title{
¿HAY INCONMENSURABILIDAD TECNOLÓGICA?
}

\author{
IS THERE TECHNOLOGICAL INCOMMENSURABILITY?
}

\author{
Daian T. Flórez Q.*
}

\begin{abstract}
Resumen
Mediante la noción matemática de inconmensurabilidad, Kuhn indicó la falta de un lenguaje común que hiciese posible la traducción de los términos que se emplean de ordinario en las teorías científicas. Sin embargo, Kuhn no extendió el alcance de la relación de inconmensurabilidad al dominio de la tecnología, aunque recurrió a él para ilustrar este fenómeno semántico. Pese a lo controversial que pueda resultar, en este trabajo mostraré que la historia de la tecnología proporciona la evidencia necesaria a favor de la tesis de que hay inconmensurabilidad tecnológica. Para ello, examinaré dicha tesis y explicaré cómo operaría en el dominio de la tecnología. También analizaré dos casos históricos a su favor. El primero proviene de la sustitución de la teoría del contacto de la pila voltaica por la teoría química de la batería. El segundo caso se da a partir del tránsito de la teoría material del calor a la termodinámica.
\end{abstract}

Palabras clave: Kuhn. inconmensurabilidad tecnológica, pila voltaica, motor de vapor, calor latente.

\begin{abstract}
By means of the mathematical notion of incommensurability Kuhn explained the lack of a common language that made it possible the translation of terms ordinarily used in scientific theories. It is clear that he did not extend the scope of this semantic relation to the domain of technology, despite taking examples of incommensurability from its quarters. Regardless of how controversial can be such a contention, in this paper I shall show that the history of technology provides the necessary evidence to support the thesis that there is technological incommensurability. To this end, I shall examine this thesis and explain how it would work in the domain of technology. I shall also analyze two historical ca-
\end{abstract}

* Doctora en Filosofía, Profesora. Universidad de Caldas, Universidad Nacional de Colombia, Colombia. E-Mail: daian.florez@ucaldas.edu.co 
ses. The first one comes from the substitution of the theory of contact or electrostatic conception of the voltaic battery by the chemical theory of the battery. The second one is the transit from the material theory of heat to thermodynamics.

Keywords: Kuhn, incommensurability, technology, voltaic battery, steam engine, latent heat.

Recibido: 2020-O5-OI

Aceptado: 2020-06-09

\section{La tesis de la inconmensurabilidad interteórica}

Kuhn presentó la tesis de la inconmensurabilidad en dos versiones que, stricto sensu, no difieren sustancialmente ${ }^{1}$. La primera versión (años sesenta), que se conoce como versión semántica, apareció en The Structure of Scientific Revolutions ${ }^{2}$ (1962/1996, en adelante SScR). La segunda versión se encuentra en una edición de varios artículos, preparada por James Conant y John Haugeland, y cuya edición final Kuhn conoció antes de su muerte. Estos artículos fueron compilados bajo el título The Road Since Structure (2000). La versión que allí aparece se conoce como versión taxonómica ${ }^{3}$. Por los propósitos que me he trazado en este artículo, aquí

I Hay quienes consideran -entre ellos, Sankey (1993) y Falguera (1998)- que es conveniente distinguir tres o cuatro fases en la formulación de la tesis de la inconmensurabilidad de teorías, respectivamente. Sin embargo, apelando al principio de parsimonia, en este trabajo suscribiré una formulación binaria de la tesis, i.e., aquella que identifica dos versiones de la tesis de la inconmensurabilidad: la versión semántica de los años 60 y la versión taxonómica de los años 80.

2 La primera formulación de la tesis de la inconmensurabilidad entre teorías separadas por una revolución científica aparece en SScR, pero también se puede encontrar en textos posteriores como el Post Scriptum de 1969, La tensión esencial y "Segundos pensamientos sobre paradigmas".

3 Una formulación esquemática de esta versión de la tesis sería: Dos teorías $T_{I}$ y T2 son inconmensurables syss sus estructuras taxonómicas no son homologables. Aunque la primera versión de la tesis de la inconmensurabilidad no es radicalmente defectuosa, la segunda tiene la virtud de explicar las causas del cambio semántico (tanto a nivel del significado, como del referente) en teorías inconmensurables. La razón de dicho fenómeno es que no puede haber homología entre las estructuras taxonómicas o de clase cuando hay inconmensurabilidad. Ahora bien, las categorías taxonómicas son términos de clase que poseen, al menos, dos propiedades: (i) están precedidos por un artículo indefinido, y (ii) deben cumplir con el principio de no solapamiento, o lo que es lo mismo, las categorías taxonómicas sólo se pueden solapar de género a especie y no de especie a especie. Kuhn explica esto a partir del ejemplo: "no hay perros que también sean gatos". No es el caso que dos de estas categorías o clases puedan tener algunos casos en común a menos que una de ellas subsuma entera y necesariamente a la otra. Las estructuras taxonómi- 
sólo me ocuparé de la versión semántica.

Kuhn utiliza por primera vez el término 'inconmensurabilidad' en el acápite de $S S c R$, titulado "The nature and necessity of Scientific Revolutions". En dicho acápite, el físico norteamericano se ocupa de mostrar la naturaleza y alcance de las revoluciones científicas, de suerte que la inconmensurabilidad es una de las consecuencias de los cambios revolucionarios, i.e., de la sustitución de un paradigma ${ }^{4}$ por otro. Tras indicar que las revoluciones científicas "son episodios de desarrollo no-acumulativo en los que un antiguo paradigma es reemplazado, completamente o en parte, por otro nuevo e incompatible" (Kuhn I996, p. 92), Kuhn anticipa, prima facie, cuatro objeciones en contra de la tesis de que hay cambios científicos dramáticos, a causa de las revoluciones científicas. En respuesta a una de estas objeciones -a la que llamaré la objeción de la reducción interteórica-, Kuhn introduce la tesis de la inconmensurabilidad.

Objeción de la reducción interteórica en contra de la tesis de que hay revoluciones científicas

De manera esquemática, podemos plantear la objeción como sigue:

(PI) Si la teoría newtoniana puede derivarse de la teoría de la relatividad de Einstein (bajo la suposición de que las velocidades relativas de los cuerpos estudiados sean inferiores a la velocidad de la luz), entonces no hay cambios dramáticos en la ciencia, tal y como lo supone la tesis de que hay revoluciones científicas.

(P2) La teoría newtoniana puede derivarse de la teoría de Einstein (bajo la suposición de que las velocidades relativas de los cuerpos estudiados sean inferiores a la velocidad de la luz).

(C) Por lo tanto, no hay cambios dramáticos en la ciencia, como lo afirman quienes mantienen que hay revoluciones científicas.

cas distintas, i.e., aquellas cuyas diferencias se dan a partir de la relación de subsunción y exclusión son inconmensurables, cuando sus diferencias dan como resultado términos con significados fundamentalmente dispares (cf. Kuhn 2000, p. 92).

4 Es bien sabido que para algunos de los críticos más conspicuos de la teoría de la ciencia kuhniana, el filósofo no logra ofrecer una definición satisfactoria del término 'paradigma' en virtud de su polisemia (cf. Masterman 1975). Sin embargo, creo que es posible ofrecer una definición funcional para los propósitos de este artículo: un paradigma se puede entender como una entidad híbrida que comprende no sólo un conjunto de teorías científicas, leyes y modelos, sino también un conjunto de compromisos ontológicos, conceptuales y metodológicos (e.g. la mecánica clásica de partículas). 
Para mostrar que el antecedente de $\left(\mathrm{P}_{\mathrm{I}}\right)$ es falso, Kuhn argumenta que no es posible reducir (o deducir) la teoría newtoniana de la teoría de la relatividad, y para ello nos pide que le sigamos en el siguiente razonamiento:

(SI) Supongamos que la teoría de la relatividad comprende los enunciados: EI, E2...En (debemos suponer que estos enunciados contienen variables y parámetros, tales como: posición espacial, tiempo, masa en reposo, etc.).

(S2) Supongamos que la teoría newtoniana comprende los enunciados: Ni, N2...Nn (debemos suponer que estos enunciados también contienen variables y parámetros, tales como: posición espacial, tiempo, masa en reposo, etc.).

(S3) Supongamos que queremos derivar Ni de Ei y N2 de E2. Pero tal derivación no es posible porque las referencias físicas no son idénticas. La masa newtoniana se conserva, la einsteniana se transforma. (Kuhn I962/I996, pp.IOI-IO2.).

De acuerdo con Kuhn, lo que imposibilita la reducción interteórica de la teoría newtoniana a partir de la teoría einsteniana es la inconmensurabilidad de teorías. El argumento es sencillo: una conditio sine qua non para la reducción interteórica es la preservación de los significados de los términos de las teorías científicas. Como esto es justamente lo que no se da -a causa de la inconmensurabilidad-, la reducción interteórica resulta lógicamente imposible. Dicho en otras palabras, no es posible derivar la teoría newtoniana de la teoría einsteniana porque estas son inconmensurables.

Esta primera versión de la tesis, tal y como Kuhn la presenta en su $S S c R$, se suele denominar versión semántica, en razón de que la inconmensurabilidad queda estrechamente vinculada al cambio semántico del significado y el referente de los términos de las teorías científicas.

Una formulación esquemática de la versión semántica de la tesis de la inconmensurabilidad sería la siguiente:

(Di) Dos teorías Ti y T2 son inconmensurables syss los términos que se usan en el lenguaje de Ti no son semánticamente equivalentes a los los términos que se usan en el lenguaje de T2.

Con base en la definición anterior, es claro que la tesis de la in- 
conmensurabilidad es una tesis de clara índole lingüística. Ahora bien, ¿bajo qué condiciones - necesarias y suficientes- dos teorías científicas son inconmensurables? Como la inconmensurabilidad es una relación diádica, i.e., es una relación entre pares de teorías sucesivas separadas por una revolución científica, podemos decir que, en rigor, dos teorías $\mathrm{T}_{1}$ y $\mathrm{T}_{2}$ son inconmensurables syss:

(i) Try T2 son teorías sucesivas separadas por una revolución científica.

(ii) Los términos que usa Ti no son semánticamente equivalentes a los términos que usa $\mathrm{T} 2$, por lo que no son intertraducibles.

Esta versión de la tesis queda ligada al fracaso de la traducción completa entre dos teorías: Kuhn entiende por intraducibilidad la imposibilidad de aplicar un mecanismo que sustituya palabras o secuencias de palabras en un idioma por palabras o secuencias de palabras en otro idioma, garantizando la intercambiabilidad salva veritates. Dicho de otra manera:

(iii) No hay un lenguaje común (Lc) que haga posible una traducción del lenguaje Li de Ti al lenguaje L2 de T2.

Para que quede más claro, la traducción es un mecanismo mediante el cual se sustituyen sistemáticamente palabras y expresiones en una lengua por palabras y expresiones en otra lengua. El éxito o fracaso de

5 En su defensa de la tesis de la inconmensurabilidad como intraducibilidad, Kuhn (2000) apela al principio de indeterminación de la traducción de Quine, según el cual, cuando traducimos un lenguaje a otro, no hay manera alguna de saber cuál es la traducción correcta de las expresiones. Ello se puede explicar a partir del célebre experimento mental de Quine en Palabra y objeto (1968) en el que plantea el siguiente escenario hipotético: supongamos que un lingüista se enfrenta a una tribu recién descubierta, para cuyo lenguaje no existe ningún manual de traducción. El lingüista podría generar dos manuales: uno escrito en su lenguaje y otro en el lenguaje de los nativos. Sin embargo, pese a que las palabras escritas en ambos manuales son compatibles con los mismos estímulos sensoriales, éstas pueden ser incompatibles entre sí. Por lo tanto, según Quine, para que sea posible una traducción radical se requiere la preservación o escrutabilidad de la referencia. Como esto es justamente lo que no se da (sin importar las conductas observables de los nativos), la consecuencia que se deriva es la existencia de múltiples traducciones incompatibles entre sí. La posición de Kuhn al respecto es diferente de la de Quine. Kuhn sostiene que una traducción debe preservar no sólo la referencia, sino también el sentido. Como el requisito para efectuar una traducción exitosa -según Kuhn- es la preservación o invariancia del significado y la referencia de los términos involucrados -y esto es precisamente lo que no se da cuando hay inconmensurabilidad-, el fracaso de la traducción es inevitable. 
dicho mecanismo radica en (a) la preservación del significado y la referencia de las palabras involucradas, y (b) en la la intercambiabilidad salva veritate. Ahora bien, (a) es una conditio sine qua non de (b); sin embargo, como a causa de la inconmensurabilidad no se preserva ni el significado ni el referente de los términos, el fracaso de la traducción es inevitable.

(iv) Como consecuencia de (ii) y (iii) no hay un criterio lógico, i.e., un algoritmo de decisión racional, con base en el cual se pueda elegir entre Ti o T $2^{6}$.

(v) Como consecuencia de (i), (ii) y (iii) ocurre un cambio en los compromisos metafísicos, ontológicos y metodológicos de las comunidades científicas?

(vi) Como consecuencia de (i), (ii) y (iii): hay inconmensurabilidad perceptual, i.e., Ti y T2 son inconmensurables perceptualmente syss los partidarios de Ti y los partidarios de T2 ven el mundo de manera diferente ${ }^{8}$.

Según Kuhn, tras una revolución científica, hay un cambio en la forma de visión del mundo o cambio perceptual que se deriva de los compromisos teóricos que suscriben los miembros de las comunidades científicas. Para apoyar este planteamiento resulta importante mostrar cómo dos observadores pueden ver simultáneamente el mismo objeto de formas distintas: "Para que estas observaciones cobren importancia filosófica, resulta crucial que los observadores reconozcan que están observando un solo objeto de formas diferentes" (Brown I984, p. 75. Énfasis añadido).

Un ejemplo que ayuda a esclarecer la tesis de que una sola cosa puede verse de formas diferentes es el cambio gestáltico. Piensen en figuras tales como las de la anciana-jóven o las de la cara-jarrón, las cuales

6 Ello no quiere decir que no dispongamos de criterios para adelantar la evaluación y elección racional entre teorías rivales. El asunto es que tales criterios no son algoritmos de decisión racional (e.g., verificación o falsación), sino que se trata más bien de criterios pragmáticos. Para un análisis más detallado remito al lector interesado a Kuhn (1998) "Objectivity, value judgment and theory choice."

7 Los compromisos metafísicos y ontológicos que suscriben los científicos -a partir de una teoría- les indica qué entidades contiene o no el universo. Los compromisos metodológicos aluden, por contraste, al tipo de reglas y prácticas admisibles para los miembros de la comunidad científica.

8 Una consecuencia adicional que se deriva de la inconmensurabilidad es el colapso en la comunicación, pero no me ocuparé de este problema en este escrito. Remito al lector interesado a La tensión esencial (1982). 
aparecen a un único observador en cualquiera de las dos formas. Las figuras reversibles, como también se les llama, proporcionan un caso claro de una situación en la que un observador ve dos cosas diferentes, mientras que el estímulo retinal sigue siendo uno solo, e.g., la célebre figura del pato-conejo. Según el ángulo hacia el cual dirija mi atención veré un pato o un conejo: si observo de derecha a izquierda veré el pato. Si observo de izquierda a derecha veré el conejo.

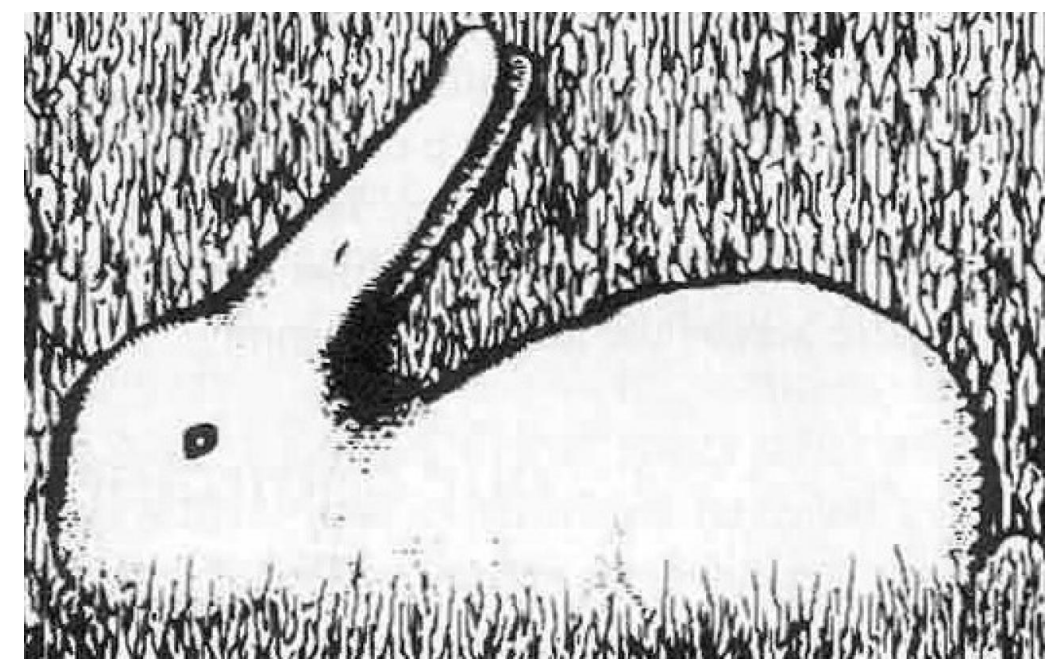

Figura I: El pato-conejo. Fuente: wikipedia.

Ahora bien, Kuhn no solo recurre a las figuras reversibles de la Gestalt para apoyar la tesis de que hay diferencias perceptivas a causa de los cambios revolucionarios, además de formular varios argumentos históricos (e.g., Lavoisier vio oxígeno allí donde Priestley vio aire deflogistizado), plantea un encuentro imaginario entre Aristóteles y Galileo a propósito de la percepción de cuerpos oscilantes que penden de un hilo, cuerda o cable: si el estagirita y el físico italiano estuviesen frente a dicho cuerpo, mientras el primero "observaría" un objeto en caída impedida hacia su lugar natural de reposo, el segundo "observaría" un péndulo, que ejemplifica de manera harto ilustrativa un caso de caída libre, reducible a regularidades expresables matemáticamente.

Por otro lado, en su defensa de la tesis de la inconmensurabilidad, Kuhn también ofrece distintos argumentos históricos. Destaco los tres más importantes: (i) el tránsito de la física aristotélica a la física newto- 
niana, (ii) el tránsito de la teoría del contacto a la teoría química de la pila voltaica, (iii) el tránsito de la química del flogisto a la química de Lavoisier. Debido a los límites que le he puesto al presente trabajo, sólo explicaré de manera sucinta (i), para indicar los cambios semánticos profundos que tienen lugar tras una revolución científica. En el segundo apartado de este artículo me ocuparé de (ii), dado que este caso histórico apoyaría, particularmente, lo que he denominado aquí como tesis de la inconmensurabilidad tecnológica.

Con base en el primer episodio histórico, Kuhn argumenta que si comparamos la cinemática aristotélica con la cinemática newtoniana, advertimos que, aún cuando ambas teorías utilizan el término "movimiento", se refieren a conjuntos de fenómenos muy disímiles entre sí. La teoría aristotélica, por ejemplo, se refiere con el término "movimiento" al cambio en general, de manera que éste incluye distintas variedades de cambio (el aumento, la disminución, el crecimiento, el envejecimiento), que incluyen, además del cambio de lugar -propio del movimiento local de objetos en el espacio-, fenómenos como la transformación de una bellota en roble, o variantes, como el paso de la enfermedad a la salud y viceversa. Adicionalmente, para el estagirita el movimiento local es un cambio de cualidad, que incluye distintas subcategorías de movimiento: el crecimiento; las alteraciones de intensidad, como el calentamiento de una barra de hierro, y aquellos cambios que tienen lugar mediante la transformación de propiedades por lo cual resulta adecuado clasificarlos bajo el concepto de proceso. Para Newton, por contraste, el movimiento se refiere únicamente a un cambio de estado.

El argumento que Kuhn ofrece a partir de estas diferencias conceptuales es, sin lugar a dudas, bastante ingenioso, ya que muestra que el progreso científico no consiste en la incorporación de una teoría con menor poder explicativo, en otra con mayor poder explicativo. En otras palabras, el progreso no consiste en que una teoría como la cinemática aristotélica sea subsumida por otra teoría, la cinemática newtoniana, ya que de hecho, el término "movimiento" en la física aristotélica designa un número más amplio y variado de cambios que su contrapartida en la física newtoniana.

Por otro lado, Kuhn argumenta también que el significado de un término científico se da de manera holista, i.e., el significado de un tér-

9 La literatura que explica las diferencias entre el movimiento local considerado como un proceso y el mismo fenómeno considerado como un estado es abundante. Remito al lector a los trabajos de Blanché (I975), Koyré (1977, 1980) y García (1997). 
mino depende del significado de otros conceptos. Así, el significado del término "movimiento" se deriva de los postulados mismos de la cosmología aristotélica y de cómo se relaciona con otros términos de su física, inter alia, los conceptos de "materia", "posición" o "lugar". Un ejemplo que ilustra la idea anterior, consiste en que, si el mundo es finito -según los postulados de la cosmología aristotélica-, los cambios en la naturaleza obedecen tanto a variaciones cualitativas como a cambios de posición (izquierda-derecha, adelante-atrás, arriba-abajo) o con respecto al lugar natural de los objetos, según la conocida doctrina de los lugares naturales y no-naturales y del movimiento natural o violento. Tal conjunto de principios explica por qué para Aristóteles la materia es imprescindible desde su perspectiva finita del mundo y por qué, como plenista que es, considera el vacío comparable a la idea de "un círculo cuadrado" (Kuhn 2000, p. 19).

Ahora bien, como no hay equivalencia semántica entre el término 'movimiento' en la cinemática aristotélica y el término 'movimiento' en la cinemática newtoniana, podemos concluir que ambas teorías son inconmensurables.

Como bien se sabe, tras la formulación de la tesis de la inconmensurabilidad de teorías surgieron numerosas y muy variadas objeciones en su contra ${ }^{\text {Io }}$. Debido a ello, el lector podría pensar que es una necedad sostener que no sólo hay inconmensurabilidad en el dominio de la ciencias empíricas, sino que también hay inconmensurabilidad en el dominio de la tecnología. Sin embargo, encuentro dos razones muy persuasivas que motivan mi planteamiento de que hay inconmensurabilidad tecnológica. La primera es que uno de los argumentos históricos que Kuhn ofrece como evidencia a favor de la tesis de la inconmensurabilidad interteórica proviene justamente del dominio de la tecnología, me refiero al tránsito de la teoría del contacto de la pila voltaica a la teoría química de la batería. La segunda razón estriba en que la historia de la tecnología nos proporciona evidencia adicional a favor de esta tesis, como lo mostraré a partir del tránsito (o sustitución) de la teoría del calor latente de Black por la termodinámica. Veámos cada uno de estos argumentos históricos.

\section{Inconmensurabilidad tecnológica}

Sostener que hay inconmensurabilidad en el dominio de la tecnología puede suscitar reacciones de rechazo aún más apasionadas de las 
que estamos acostumbrados a encontrar en el terreno de las ciencias empíricas, por dos razones. En primer lugar, se suele identificar a la tecnología con la totalidad de los artefactos que son el resultado de las acciones y procesos tecnológicos (Kline 2003) ${ }^{\mathrm{II}}$, de suerte que si la inconmensurabilidad es una tesis semántica, no resulta atribuible -o predicable- a objetos como los artefactos. En segundo lugar, hay una creencia generalizada según la cual no hay teorías tecnológicas. De manera que así como la ciencia es una actividad fundamentalmente teorizadora, la tecnología -en tanto esencialmente práctica- se caracteriza por la creación de artefactos. Con todo, no es difícil mostrar que las creencias anteriores están lejos de ser indisputables. Pero antes de acometer esta tarea, consideremos un argumento que podrían esgrimir aquellos que se resisten a admitir que hay inconmensurabilidad tecnológica:

(PI) Si los productos tecnológicos son artefactos (y los artefactos qua objetos materiales no tienen propiedades semánticas, e.g., ser inconmensurables), entonces no hay inconmensurabilidad tecnológica.

(P2) Los productos tecnológicos son artefactos.

(C) Por lo tanto, no hay inconmensurabilidad tecnológica.

Sin embargo, un argumento así fallaría porque termina identificando la tecnología con la suma de los artefactos que son el resultado de un conjunto de acciones muy complejas ${ }^{12}$. Si bien es cierto que, desde la perspectiva de sentido común, los artefactos constituyen nuestro encuentro inmediato con la tecnología, la tecnología es mucho más compleja que lo que han pensado de ordinario los filósofos. De hecho, la tecnología involucra prácticas convencionales como diseñar -experimentar, calcular, simular- y desarrollos tecnológicos como los artefactos (o entidades, mucho más difíciles de clasificar, como los programas de computación -software-). En este argumento que proviene de la perspectiva del sentido común, se incurre, de hecho, en un error de razonamiento, comparable al que cometeríamos si identificáramos simpliciter la ciencia con

II Kline encuentra cuatro usos del término 'tecnología'. El primero de ellos identifica a la tecnología con la totalidad de los artefactos: "HARDWARE (OR ARTIFACTS): Possible denotation: non-natural objects, of all kinds, manufactured by humans" (Kline 2003, p. 210).

I2 En este trabajo no intentaré ofrecer una definición no-controversial de 'tecnología'. Este problema es rico y complejo, como le he mostrado en Florez et al. (2019). 
las leyes o las teorías científicas. Si bien es cierto que entre los productos -o resultados- más notables de la actividad científica se encuentra la formulación de leyes y teorías, la ciencia es mucho más que eso. Como actividad compleja, la ciencia también involucra prácticas (e.g., contrastar, medir, calcular, experimentar) (cf. Díez \& Moulines 1999).

El error de razonamiento que se puede identificar en el argumento anterior configuraría una falacia de composición. Recordemos que este error consiste en transferir una propiedad de las partes al todo, o un atributo de los miembros de un grupo, al grupo, e.g., cada una de las partes de esta máquina está bien hecha, por lo tanto, esta máquina (como un todo) está bien hecha. En este ejemplo se transfiere ilegítimamente propiedades de la parte, particularmente de las piezas de las que se predica que están bien hechas, al todo, por lo cual se concluye que toda la máquina está bien hecha. En el argumento desde la perspectiva del sentido común para rechazar que hay inconmensurabilidad tecnológica se incurre en este mismo error de razonamiento pues se transfiere una particularidad de uno de los productos de la tecnología, la imposibilidad de atribuir a objetos (en este caso a los artefactos) propiedades semánticas (como por ejemplo, ser inconmensurable), al todo, y de esta movida se extrae la conclusión de que siempre es erróneo predicar la inconmensurabilidad de la tecnología.

Adicionalmente, no es cierto que no haya teorías en la tecnología. Por ejemplo, en el dominio de la ingeniería estructural (una rama de la ingeniería civil que se ocupa del diseño estructural aplicable a construcciones tales como edificios, puentes, muros de contención, entre otros) se han formulado, inter alia, la teoría de vigas y la teoría de placas o láminas.

Es bien sabido que el ingeniero estructural tiene entre sus propósitos el diseño de estructuras seguras. El éxito de una tarea como ésta, ha sido posible gracias a que físicos e ingenieros han llevado a cabo un análisis teórico del comportamiento de las estructuras. Una de las teorías más reputadas para el análisis de estructuras es precisamente la teoría clásica de vigas que fue formulada en el siglo XVIII por los matemáticos y físicos Leonhard Euler y Daniel Bernoulli ${ }^{13}$. La teoría de vigas juega un rol importante en el análisis estructural, dado que proporciona al ingeniero una herramienta simple para analizar estructuras muy variadas. Se suele usar en la etapa de prediseño ya que proporciona información valiosa sobre el comportamiento de las estructuras (cf. Bauchau \& Craig 2009).

I3 Aunque no es la única disponible en ese campo. El lector puede remitirse a la teoría de vigas más reciente formulada por el ingeniero ucraniano Timoshenko. 
A partir de la teoría de vigas es posible calcular no sólo las tensiones a las que se somete una viga, sino también sus desplazamientos. Es importante llevar a cabo dicho cálculo ya que con base en él se examina la resistencia de los materiales usados en la construcción ${ }^{14}$. Por otra parte, su uso no se restringe al dominio de la ingeniería civil, ya que muchas partes de algunas máquinas son estructuras en forma de vigas: brazos de palanca, ejes, etc. Algunas estructuras aeronáuticas, como las alas y el fuselaje, también pueden tratarse como vigas de pared delgada (cf. Bauchau \& Craig 2009) ${ }^{15}$.

De manera que, si una conditio sine qua non para que se de la inconmensurabilidad es que haya teorías, y en algunas subáreas de la tecnología, como la ingeniería, hay teorías, entonces la tesis de que hay inconmensurabilidad tecnológica es prima facie admisible ${ }^{16}$. Ahora bien, para mostrar que en el dominio de la tecnología se satisfacen las condiciones necesarias y suficientes que señalé en los numerales (i) a (vi), veamos los siguientes dos argumentos históricos: el tránsito de la teoría del contacto de la pila voltaica a la teoría química y el tránsito de la teoría material del calor a la termodinámica.

\section{El tránsito de la teoría del contacto de la pila voltaica a la teoría electroquímica}

Con seguridad, todos nosotros hemos utilizado para propósitos muy variados las pilas eléctricas. Algunos de ustedes seguramente sabrán que una pila eléctrica es un dispositivo que transforma energía química en energía eléctrica. Finalmente, sólo unos pocos familiarizados con la historia de la tecnología sabrán que su inventor fue el químico y físico italiano Alessandro Volta. Kuhn presenta este acontecimiento histórico así:

I4 De hecho, la teoría de vigas hace parte del campo dedicado al estudio de la resistencia de materiales, que ocupa no sólo a los ingenieros civiles (especialmente a los estructurales), sino también a los ingenieros mecánicos e ingenieros industriales.

I5 Un argumento adicional se podría esgrimir a partir del estudio de la resistencia de materiales. Allí podemos recurrir a otra teoría propia de la ingeniería estructural como la teoría de placas, que fue formulada por el ingeniero mecánico Raymond Mindlin (195I) y el ingeniero civil Reissner (1945). Ambos dedicaron sus mejores esfuerzos al estudio de la deformación de placas y láminas hasta formular un conjunto de hipótesis cinemáticas sobre cómo se deforma una placa o una lámina bajo flexión.

I6 El lector podría pensar que mi argumento depende de mostrar que la ingeniería es una tecnología (o constituye una de las ramas de la tecnología) y ésta es una afirmación que se podría controvertir. Sin embargo, pido al lector que admita, por ahora, que la ingeniería es una subárea de la tecnología, tal y como lo admiten también Bunge (1980), Jarvie (1967), Feibleman (1983) y Skolimowski (I966). 
El año I80o es notable, entre otras cosas, por ser el año en el que Volta descubrió la batería eléctrica. El descubrimiento fue anunciado en una carta dirigida a Sir Joseph Banks, presidente de la Royal Society. La carta iba destinada a la publicación e iba acompañada por la ilustración reproducida aquí como figura (I). Desde un punto de vista moderno hay algo peculiar en ella, si bien incluso los historiadores raramente notan esta peculiaridad. Mirando a cualquiera de las llamadas "pilas" (de monedas) en la parte inferior del diagrama, se ve leyendo hacia arriba desde la parte inferior derecha, un disco de zinc, $\mathrm{Z}$, luego un disco de plata, A, después un trozo de papel secante humedecido, a continuación un segundo disco de zinc, y así sucesivamente. (Kuhn 2000, pp. 20-2I).

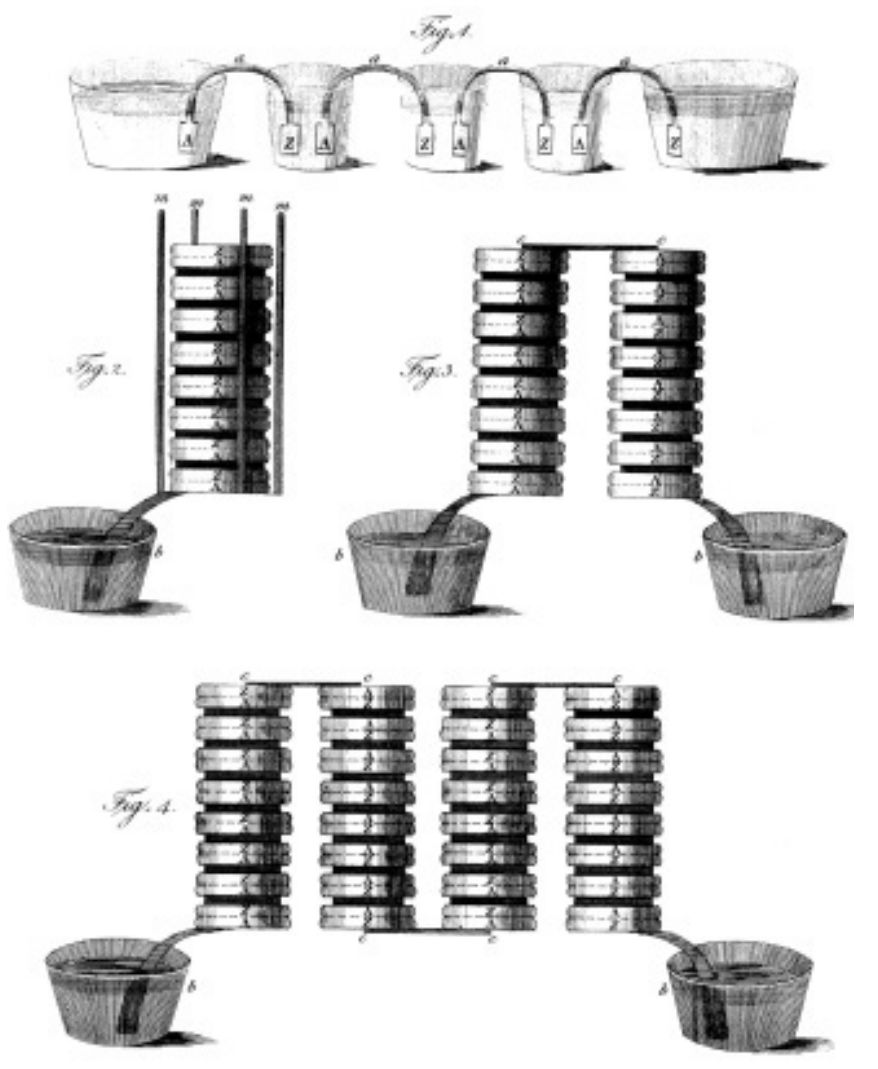

Figura 2. Fuente: wikipedia. Esta "Figura 2" es la que Kuhn describe en su texto como "Figura I".

La pila voltaica se compone de una serie de discos puestos unos sobre otros en el siguiente orden: un disco de cobre, otro de zinc y plata a los que se superpone un papel secante humedecido (por lo general 
impregnado en salmuera) todos ellos apilados. El ciclo zinc, plata, papel secante humedecido se repite un número entero de veces, ocho para ser fieles a la figura original de Volta. Justamente por esta disposición de sus componentes, éste dispositivo toma el nombre de "pila" que ha conservado hasta nuestros días.

Volta creyó que la corriente eléctrica que producía la pila se debía al contacto entre los materiales que la componían. Es por ello que a dicha explicación teórica se le conoce como "la teoría del contacto" ${ }^{17}$. Sin embargo, la teoría del contacto de Volta no tardó en ser combatida por muchos físicos, entre ellos, por su coetáneo y compatriota Giovanni Fabbroni, quien tras observar que los discos de zinc se oxidaban al contacto con el papel humedecido, pensó que la oxidación -o corrosión- era la causa principal de la electricidad. Esto sugirió que la corrosión era realmente parte integrante de la capacidad de la batería para producir una corriente y condujo a la sustitución de la teoría de la tensión del contacto de Volta por la teoría electroquímica defendida por Fabbroni, Wollaston, Davy, M. de la Rive, Antoine Becquerel, para quienes la fuerza electromotriz de la pila se da como resultado de una reacción química ${ }^{18}$.

Como es de esperar, las diferencias explicativas que acabamos de resumir conducen, en opinión de Kuhn, a divergencias semánticas profundas. Entre ellas se destaca la emergencia de los dos términos que muestran por qué la teoría del contacto y la teoría electroquímica de la pila eléctrica serían inconmensurables: los términos de "elemento de batería" y de "resistencia eléctrica".

Mientras un elemento unidad (o elemento de la batería como tam-

I7 Remito al lector interesado a la siguiente carta de Volta en la que anuncia y explica el funcionamiento de su pila: "On the electricity excited by the mere Contact of conducting substances of different Kinds. In a letter from Mr. Alexander Volta, F.R.S. Professor of Natural Philosophy in the University of Pavia to the Rt. Hon. Sir Joseph Banks, Bart. K.B. P.R.S. June 26, I80o".

I8 Antoine Becquerel fue el primero en emplear un proceso electrolítico para separar el metal del mineral en el que se halla incrustado. La electrólisis es un procedimiento que consiste en hacer pasar una corriente eléctrica a través de determinadas substancias conductoras (o electrolitos). Este descubrimiento le convirtió en el padre de la electroquímica. Otro de sus grandes hallazgos, realizado en 1829 , fue la pila de corriente constante, explotada años después por el químico británico John Frederic Daniell (1790-1845). Por este y otros inventos y descubrimientos de suma importancia para el desarrollo de la ciencia contemporánea, fue nombrado miembro de la Academia de Ciencias en I829, y honrado, ocho años después, con el prestigioso cargo de catedrático de Física del Muséum d'Histoire Naturelle (Museo de Historia Natural) de París, puesto en el que habrían de sucederle su hijo y su nieto. 
bién se conoce) corresponde, en la teoría de contacto de Volta, a la siguiente disposición: un disco de zinc unido a uno de plata (poniendo en contacto los metales) a los que se les superpone un trozo de papel secante humedecido (Kuhn 2002, p. 32), un elemento de batería, desde una perspectiva actual pero similar a la de la pila voltaica, se compone de un disco de zinc, un disco de plata y entre estos se pone el papel secante humedecido. Esta nueva disposición de los elementos, que corresponde a la concepción moderna, implica la inversión del flujo de la corriente, además de hacerla externa, puesto que en el sistema de Volta es interna (Kuhn 2002, p. 34). Según Kuhn:

Mucho más importante conceptualmente es el cambio en la fuente de corriente efectuado por la transición. Para Volta la superficie entre los dos metales era el componente esencial del elemento de la batería y, necesariamente, la fuente de la corriente que el elemento producía. Cuando el elemento fue puesto al revés el líquido y sus dos superficies de contacto con los metales proporcionaron los elementos esenciales, y los efectos químicos en estas superficies pasaron a ser la fuente de la corriente. (Kuhn 2000, pp. 22-23).

En el tránsito de la teoría del contacto de la pila voltaica a la teoría electroquímica no sólo cambia el significado del término "elemento de unidad" en razón de que cambia la disposición de los elementos que la integran, también cambia el significado del término "resistencia eléctrica”. Según sus argumentos:

En un material aislante de una sección transversal dada, la resistencia se medía por la longitud más corta que el material podía tener sin permitir el paso de la corriente o tener pérdidas -dejando de aislar- cuando era sometido a un voltaje dado.

En un material conductor de una sección transversal dada, la resistencia se medía por la longitud más corta que el material podía tener sin fundirse cuando era conectado a un voltaje dado. (Kuhn 2000, p. 24).

Nótese entonces que, a la luz de la teoría del contacto, la resistencia eléctrica se medía con base en la longitud más corta que debía tener el material para impedir el paso de la corriente, o que debía tener para no fundirse. Si hoy quisiésemos medir la resistencia así, los resultados serían, en opinión de Kuhn, incompatibles con la ley de Ohm, que concibe a la batería como un modelo hidrodinámico.

En la teoría electroquímica de la pila, la expresión "resistencia eléctrica" se refiere a la oposición al flujo de corriente eléctrica a través de un 
conductor. Para medirla, los electroquímicos aprovecharon los estudios y experimentos que Georg Simon Ohm adelantó, y con los que pudo establecer -27 años después de que se inventó la pila eléctrica- la relación entre la tensión eléctrica, la corriente eléctrica y la resistencia eléctrica. Ohm descubrió el principio científico con base en el cual se puede calcular la resistencia, y que en todo caso representa una manera radicalmente distinta de cómo se medía o calculaba la resistencia eléctrica a partir de la teoría del contacto ${ }^{19}$. Para los electroquímicos, la resistencia de un conductor es directamente proporcional a su longitud (aumenta conforme es mayor su longitud) y es inversamente proporcional a su sección transversal (disminuye conforme aumenta su grosor o sección transversal).

Con base en el caso histórico anterior, podemos inferir que la teoría del contacto de la pila voltaica y la teoría electroquímica de la batería son dos teorías genuinamente inconmensurables en el dominio de la tecnología, en virtud de que los términos "elemento de unidad" (o "elemento de batería") y "resistencia eléctrica" no son semánticamente equivalentes, y en consecuencia, no son intertraducibles.

A este argumento se podría oponer la objeción de que tanto la teoría del contacto, como la teoría electroquímica de la batería son teorías científicas y no teorías tecnológicas. A lo que se puede responder que, puesto que ambas teorías se formularon con el propósito de establecer cuál era el agente causal de la fuerza electromotriz, y con ello establecer el principio de funcionamiento del dispositivo, sí que se pueden estimar como genuinas teorías tecnológicas. Dicho en otras palabras, las teorías tecnológicas son, al igual que las teorías científicas, teorías explicativas; pero, a diferencia de estas últimas, tienen un elemento característico y es que los principios explicativos en tecnología son principios de funcionamiento, tal y como lo veremos enseguida a partir del caso histórico de la explicación del funcionamiento de la máquina de vapor.

\section{El tránsito de la teoría material del calor latente a la termodinámica}

La máquina de vapor, que es el logro más emblemático de la primera Revolución Industrial, fue inventada y patentada por el ingeniero mecánico James Watt en I769. A diferencia de la pila voltaica, cuyo diseño

I9 Para un conductor de tipo cable, la resistencia está dada por la siguiente fórmula: Donde $\boldsymbol{\rho}$ es el coeficiente de proporcionalidad o la resistividad del material, $\ell$ es la longitud del cable y $\mathbf{S}$ el área de la sección transversal del mismo. 
fue explicado a partir de la teoría del contacto formulada por su propio inventor, tanto filósofos como historiadores consideran que el diseño de la máquina de vapor fue posible sin el beneficio de la ciencia. La creencia generalizada sugiere que la invención de la máquina de vapor resultó más bien de todo un proceso de experimentación y mejoramiento de los prototipos que le precedieron (las máquinas atmosféricas), por lo que su desarrollo tuvo lugar en función de la eficiencia, aún cuando la noción de eficiencia termodinámica no se había formulado explícitamente. Recordemos que Sadi Carnot -quien es considerado el padre de la termodinámica- publicó sus Reflexiones de la fuerza motriz del fuego en I824 (55 años después de patentada la máquina) y que casi un siglo después Clausius y Lord Kelvin formularon la primera y la segunda ley de la termodinámica.

Con base en lo anterior, algunos filósofos e historiadores sostienen que fue realmente el diseño de la máquina lo que favoreció el desarrollo de la ciencia, particularmente, la formulación de la teoría termodinámica: "Carnot descubrió la ciencia pura de la termodinámica como resultado de sus esfuerzos por mejorar la eficiencia del vapor" (Feibleman 1983 , p. 39). Justamente, por ello es común encontrar en la literatura metateórica la afirmación de que "es más lo que la ciencia le debe a la máquina de vapor, que lo que la tecnología le adeuda a la ciencia" (cf. Don Idhe 2013, p. 54) o en palabras de Derry \& Williams:

la máquina de vapor convierte la energía térmica en energía mecánica, y su rendimiento se rige por las leyes de la termodinámica. Estas leyes, no obstante, no fueron establecidas hasta mediados del siglo XIX, y por lo tanto, hasta entonces, en ningún modo había sido entendida la naturaleza del calor: esta circunstancia nos recuerda una vez más que tecnología no es, en modo alguno, sinónimo de ciencia aplicada. (Derry \& Williams I977, pp. 494-495)

Si bien es cierto que el diseño de la máquina de vapor precede por décadas a la teoría científica que explica -de manera sistemática- su funcionamiento, la creencia ampliamente extendida de que Watt inventó la máquina de vapor sin echar mano de ningún recurso teórico parece falsa.

Watt fue contratado en la Universidad de Glasgow en 1757 como ingeniero y allí conoció al célebre químico Joseph Black, reconocido en la historia de la química por su descubrimiento del calor latente. Watt y Black mantuvieron una relación constante hasta que este último se mudó a Edinburgh en I766. Aunque Watt insistió en que había llegado a las mismas conclusiones que Black de manera independiente, lo cierto 
es que Black no sólo ejerció un fuerte influjo en su pensamiento, sino que además le asesoró -a través de abundante correspondencia de la cual hay registros- en el diseño y montaje de muchos de los experimentos que Watt realizó en el tiempo previo a la invención del condensador de la máquina de vapor.

Con base en los registros históricos que acabo de mencionar, cabe preguntarnos: ¿cuáles fueron los recursos teóricos que sirvieron de base para el diseño e invención de la máquina de vapor? Watt estaba firmemente comprometido con la teoría material del calor. Aunque ésta es una teoría aún más arcaica -o primitiva- que la misma teoría del calórico, la teoría material del calor planteaba que el calor era una sustancia química (Miller 2009, p. 44). Dicha teoría también postulaba la existencia del calor latente. La terminología asociada a este concepto fue introducida por Black para explicar los cambios de estado (e.g., líquido a gaseoso).

Sin embargo, tal y como lo plantea Miller en su estupendo libro James Watt, Chemist, Watt exploró los alcances de esta idea mucho más allá de lo que lo hiciera el propio Black (cf. Miller 2009, p. 45). Tanto así que consiguió formular la bien conocida Ley de Watt la cual estipula que la suma del calor latente y sensible es una constante.

Watt no sólo usó esta idea en su desarrollo del 'trabajo expansivo' de la máquina de vapor, sino que también, y de un modo aún más importante, en sus ideas sobre la transformación del agua en aire. (Miller 2009, p. 45)

A partir de estos elementos de juicio, resulta sorprendente que filósofos de la tecnología, e incluso muchos historiadores nos hayan ofrecido una imagen - casi distorsionada- no sólo del diseño de la máquina como resultado de la aplicación del método de ensayo-error, en total ausencia de recursos teóricos, sino de Watt no como un hombre de ciencia, sino como un mecánico muy ingenioso:

Nos hemos predispuesto a considerar que Black habita el mundo de la "teoría" y Watt el de la "práctica". Se nos alienta a ver a Black como una figura cosmopolita de la Ilustración escocesa y a Watt como un ingenioso ingeniero colgado de sus faldas, en una relación que implica una 'transferencia de intelecto a la artesanía’. Esto es un error. (Miller 2009, p. 89)

Lo cierto es que tal vez los logros de Watt en el dominio de la ingeniería mecánica terminaron por opacar sus contribuciones en el dominio de la química que, en retrospectiva, resultan en un química muy arcaica que nos hizo olvidar -como bien lo reconoció el ingeniero An- 
drew Ure- "que la máquina de vapor fue el triunfo más espléndido que se erigió sobre la ciencia del calórico" (Miller 2009, p. 7I).

Ahora bien, supongamos que el lector concede que Watt logra explicar a partir de una teoría (i.e., la teoría material del calor -y con ella la teoría del calor latente-) el funcionamiento de la máquina de vapor. ¿Cómo apoya todo ello la tesis de que hay inconmensurabilidad en tecnología?

Tal y como lo vimos en el apartado anterior, la inconmensurabilidad es una relación diádica e interteórica; es decir, es una relación semántica que se da entre pares de teorías. Las teorías tecnológicas en disputa en el argumento histórico de la pila eléctrica son la teoría del contacto de Volta $v s$. la teoría electroquímica de la batería, en cuyo tránsito tienen lugar cambios semánticos profundos. En el argumento histórico que ofrezco a partir del diseño de la máquina de vapor, las teorías en conflicto son la teoría material del calor de Black-Watt $v$ s. la termodinámica ${ }^{20}$. Al comparar ambas teorías, el significado del término "calor latente" es diametralmente opuesto:

Estamos acostumbrados a considerar a Black como "el descubridor del calor latente", y con esto usualmente queremos decir el descubridor de lo que entendemos por el término "calor latente". Pero esto es incorrecto. Nuestro concepto de calor latente se apoya en la teoría cinética del calor; el de Black se basó en un material (químico) uno en el cual el calor latente era intrínseco a las reacciones químicas y estaba compuesto de materia ordinaria. (Miller 2009, p. 96).

Como bien lo indica Miller en este pasaje, mientras que por el término "calor latente" hoy nos referimos a la cantidad de energía que se requiere para que tenga lugar un cambio de estado en la materia, e.g., de estado sólido a líquido o de líquido a gaseoso, Black y Watt entendían el calor latente como una propiedad química intrínseca de la materia ordinaria. De hecho, Black había interpretado "el calor latente" como la causa de los cambios de estado de la materia: el agua se convertía en vapor, por ejemplo, por el calor adicional que tenía que ser suministrado, y que no era "detectable por el termómetro" (razón por la cual lo llamó "calor latente" o calor oculto) y que se combinaba con la materia del agua para producir vapor (cf. Miller 2009, p. 45).

20 "My immediate concern, however, is to emphasize the fact that from I764 Watt's work on heat was undertaken as part of Black's tradition of work. Black always made prominent reference to Watt's experiments in his lectures at Edinburgh University so far as we can rely on Robison's edition of Black's lectures" (Miller 2009, p. 94). 
Esta no es la única razón por la cual el significado del término "calor latente" en la teoría material del calor no es semánticamente equivalente al significado del término correspondiente en la termodinámica. Mientras el calor latente es -a la luz de la termodinámica- una magnitud física ${ }^{21}$ que indica la cantidad de energía térmica que una masa debe perder o absorber para cambiar de estado físico, el calor latente en la teoría de Black-Watt es una reacción química. De manera que, aún cuando el calor latente es el agente causal que explica los cambios de estado en ambas teorías, las propiedades son radicalmente diferentes: mientras que en la teoría material del calor, el calor latente es una propiedad de la materia, en la termodinámica el calor latente es energía que se transforma. Por eso no es gratuito que la teoría que Black y Watt suscribieran se denominara teoría material del calor. Para ambos, el calor tiene una naturaleza material (no dinámica, como sucede en la termodinámica actual). De hecho, Black llega a esta conclusión a partir del siguiente experimento:

El descubrimiento más importante de Black fue la observación de que el hielo que se derrite absorbe calor sin cambiar la temperatura. A partir de este reconocimiento, llegó a la conclusión de que el hielo necesita calor latente para esta modificación de la condición física. Esta fue para él la prueba sustancial de la naturaleza material del calor y en I779 uno de sus alumnos, William Cleghorn (1754-1783), formuló la definición precisa del calórico. (Bozsaky 20ı, p. 6)

Contra el argumento anterior, se podría objetar que la termodinámica no es una teoría tecnológica, por lo que apelar a este caso histórico no parece afortunado, ya que serviría más bien al propósito de defender que hay inconmensurabilidad entre teorías en el dominio de las ciencias empíricas. Sin embargo, a una objeción de esta naturaleza podríamos responder que, en efecto, aunque es indiscutible que la termodinámica pertenece al dominio de la física, la teoría material del calor que Watt defendió -y su particular manera de entender la naturaleza del calor latente- buscaba explicar fundamentalmente el funcionamiento de la máquina de vapor, que es lo propio de las teorías en tecnología. Si esto es correcto, el ejemplo que aquí examino bien podría ilustrar un caso de inconmensurabilidad entre una teoría tecnológica, a saber, la teoría material del calor y una teoría científica, i.e., la termodinámica que, en

2I Que se mide en el sistema internacional de medida en $\mathrm{J} / \mathrm{kg}$, y se representa por la letra $\mathrm{L}$. 
todo caso, se formuló tras el intento de las mejores mentes por explicar el funcionamiento de los motores de combustión externa. Adicionalmente, nada impide que reformulemos la condición (i) -estipulada en el primer acápite- y que indica que una conditio sine qua non para considerar a dos teorías $T_{1}$ y $T_{2}$ como teorías genuinamente inconmensurables estriba en que $T_{1}$ y $T_{2}$ tienen que ser teorías sucesivas separadas por una revolución científica. Podríamos muy bien demandar que $T_{\text {I }}$ y $T_{2}$ sean genuinamente rivales, i.e., que no sean trivialmente inconmensurables, o que no satisfagan la siguiente condición:

Dos teorías $T_{1}$ y $T_{2}$ son trivialmente inconmensurables syss $T_{1}$ y $T_{2}$ no tienen nada que ver entre sí; es decir, tanto su lenguaje teórico como su lenguaje contrastacional son semánticamente incompatibles. Por ejemplo, la teoría del catastrofismo en geología y la teoría del valor en la economía de Marx serían trivialmente inconmensurables. La trivialidad estriba en que no se ocupan de lo mismo o no explican los mismos fenómenos.

En las páginas anteriores argumenté que hay inconmensurabilidad tecnológica no obstante la resistencia que se podría derivar de la idea de que la inconmensurabilidad como fenómeno semántico no es atribuible a los artefactos. Un razonamiento como ese proviene de la identificación equivocada de la tecnología con los artefactos, y en la medida en que explico la naturaleza errónea de dicha identificación, considero que se puede rechazar dicha línea de análisis. Por otra parte, en esta clase de argumentos también subyace la creencia falsa de que no hay teorías tecnológicas. Dicha creencia se puede rebatir felizmente señalando, tal y como lo hice aquí, unas cuantas teorías tecnológicas, entre ellas, justamente la teoría del contacto de Volta que explica el funcionamiento de la pila o la teoría material del calor que explica el funcionamiento de la máquina de vapor.

\section{Referencias bibliográficas}

Bauchau O.A., Craig J.I. (2009) "Euler-Bernoulli beam theory". En Bauchau, O.A., \& Craig, J.I. (eds.) Structural Analysis. Solid Mechanics and Its Applications, vol I63, I73-22I. Dordrecht: Springer.

Blanché, R. (1975). El método experimental y la filosofía de la física. México: F.C.E.

Bozsaky, D. (2010). "The Historical Development of Thermodynamics". Acta Technica Jaurinensis, Vol. 3. No. I: 3-17. 
Brown, H. I. (1984). La nueva filosofía de la ciencia. Madrid: Tecnos.

Davidson, D. (200I). "On the Very Idea of a Conceptual Scheme". En Inquiries into Truth and Interpretation, I83-198. Oxford: Clarendon Press.

Derry T.K. \& Williams, T.I. (1977). Historia de la tecnología: Desde I750 hasta 190o. Volumen 2. México: Siglo XXI.

Díez, J. A. y Moulines, C.U. (1999). Fundamentos de Filosofía de la ciencia. Barcelona: Ariel.

Falguera, J. L. (1998). "Inconmensurabilidad y ontosemántica representacional". Theoria. An International Journal for Theory, History and Foundations of Science I3 (3I), I6I-I85.

Feibleman, J. K. (1983). "Pure science, applied science, and technology: an attempt at definitions". En Mitcham, C. and Mackey, R. (eds.), Philosophy and Technology: Readings in the Philosophical Problems of Technology. New York: The Free Press.

Florez, D. T. et al. (2019). “Is technology (still) applied science?” Technology in Society 59. https://doi.org/IO.IOI6/j.techsoc.2019.IOII93

García, C. E.(1997).Evolución histórica del pensamiento científico. Manizales: Universidad de Manizales.

Ihde, D. (2013). “Technology and science”. En Olsen, J.K.B., Pedersen, S.A., Hendricks, V.F. (eds.), A Companion to the Philosophy of Technology. Massachusetts: Wiley-Blackwell.

Jarvie, I. (1967). "Technology and the Structure of Knowledge". En Philosophy of Technology: Readings in the philosophical problems of technology. Mitcham \& Mackey (ed)., New York: The Free Press.

Kitcher P. (1978). "Theories, Theorists, and Theoretical Change". Philosophical Review, (87). 519-547.

Koyré, A. (1977). Estudios de historia del pensamiento científico. México: Siglo XXI.

(I980). Estudios Galileanos. México: Siglo XXI.

Kline, S. J. (2003). What is Technology? Bulletin of Science, Technology \& Society. Reprinted in: Scharff, Robert C. \& Val Dusek (eds), Philosophy of Technology: The technological condition. An Anthology. Massachusetts: Blackwell Publishing.

Kuhn, T. S. (1982). La tensión esencial. México: F.C.E. (1962/1996). The Structure of Scientific Revolutions. Chicago: The University of Chicago Press.

(1998). "Objectivity, value judgment and theory choice". En Klemke, et.al. (eds). Introductory Readings in the Philosophy of Science. New York: Prometheus Books. 
(2000). The road since structure. Chicago: The University of Chicago Press.

Masterman, M. (I975). "La naturaleza de los paradigmas". En I. Lakatos y A. Musgrave (eds.), La crítica y el desarrollo del conocimiento, 159-202. Barcelona: Grijalbo.

Miller, D. (2009). James Watt, Chemist: Understanding the origins of the steam age. Science and Culture in the Nineteenth Century. London: Pickering \& Chatto.

Popper, K. (1975). "La Ciencia Normal y sus peligros". En La crítica y el desarrollo del conocimiento, I49-I58. Barcelona: Grijalbo.

Putnam, H. (1988). Razón, verdad e historia. Madrid: Tecnos.

Quine, W. O. (1968). Palabra y objeto. Barcelona: Labor.

Sankey, H. (1993). "Kuhn's Changing Concept of Incommensurability". The British Journal for the Philosophy of Science, 44(4): 759-774.

Skolimowski, H. (1966). "The structure of thinking in technology". En C. Mitcham \& R. Mackey (eds.). Philosophy of Technology: Readings in the philosophical problems of technology. The Free Press, New York. 